\title{
Sarcoidosis presenting pulmonary subsolid nodules that mimic lung adenocarcinoma in a patient with history of uveitis and arrhythmia: a case report
}

\author{
Hye Jin Lee ${ }^{1}$, Hyewon Seo ${ }^{1}$, Seung Ick Cha ${ }^{1}$, Tae In Park ${ }^{2}$, Chang Ho Kim ${ }^{1}$, Jaehee Lee ${ }^{1}$ \\ ${ }^{1}$ Department of Internal Medicine, ${ }^{2}$ Department of Pathology, School of Medicine, Kyungpook National University, Daegu, Republic of Korea \\ Correspondence to: Jaehee Lee, MD. Department of Internal Medicine, Kyungpook National University, School of Medicine, 680 Gukchaebosang-ro, \\ Jung-gu, Daegu 700-842, Republic of Korea. Email: jaelee@knu.ac.kr.
}

\begin{abstract}
Sarcoidosis is an idiopathic systemic granulomatous disorder that can involve any organ, although the lung is the most commonly affected site; moreover, it may affect multiple organs simultaneously or serially over a long time span. Diagnosing sarcoidosis can be a challenge in cases presenting an isolated extra-thoracic lesion at the early stage of disease. Pulmonary nodular lesion, a rare radiologic finding, may also lead to delayed diagnosis of sarcoidosis. We reported a case of atypical pulmonary nodular sarcoidosis that was suspected as lung adenocarcinoma, which was diagnosed about 20 years after initial isolated extrathoracic manifestation occurred.
\end{abstract}

Keywords: Sarcoidosis; pulmonary; subsolid nodule; atypical; extra-thoracic

Submitted Jun 05, 2019. Accepted for publication Aug 21, 2019.

doi: 10.21037/atm.2019.08.98

View this article at: http://dx.doi.org/10.21037/atm.2019.08.98

\section{Introduction}

Sarcoidosis is an idiopathic granulomatous inflammatory disorder that can involve various organs $(1,2)$; moreover, it can affect multiple organs simultaneously or serially over a long time span. The diagnosis of sarcoidosis can often be missed in cases presenting involvement of an isolated uncommon site at the early stage of disease.

Radiologic manifestations of thoracic sarcoidosis, the most common presentation, have a wide spectrum $(3,4)$. Typical radiologic findings including symmetric hilar and mediastinal lymphadenopathy favor a diagnosis of sarcoidosis, while atypical radiologic findings such as single nodular lesion or lymphadenopathy at unusual locations may cause delayed diagnosis. Herein, we present a case of atypical pulmonary nodular sarcoidosis that was suspected as malignancy in a patient whose isolated extrathoracic presentation such as bilateral uveitis and complete atrioventricular (AV) block resulted in delayed diagnosis of sarcoidosis.

\section{Case presentation}

A 64-year-old woman who was an ex-smoker of 5 PYs, presented with pulmonary nodular lesions that were incidental findings on chest radiograph. She had lost her sight due to bilateral panuveitis of unknown cause 20 years ago and undergone pacemaker insertion due to complete AV block 10 years ago. In August, 2016, nodular opacity in the right upper-lung field was newly found on the chest radiograph during regular pacemaker check-up at the Department of Cardiology (Figure 1).

Chest computed tomography (CT) revealed two subsolid nodules (size, 1 and $2.7 \mathrm{~cm}$ respectively) in the right upper lobe (RUL) (Figure $2 A, B$ ) and several benign-looking mediastinal lymph nodes enlargement (Figure 2C). Interval growth of the nodules was observed on the 15 -month follow-up chest CT scan, suggesting slowly growing malignancy such as early adenocarcinoma (Figure 2D,E). There was no significant change in size of mediastinal lymph nodes (Figure 2F). ${ }^{18} \mathrm{~F}$-fluorodeoxyglucose (FDG) 


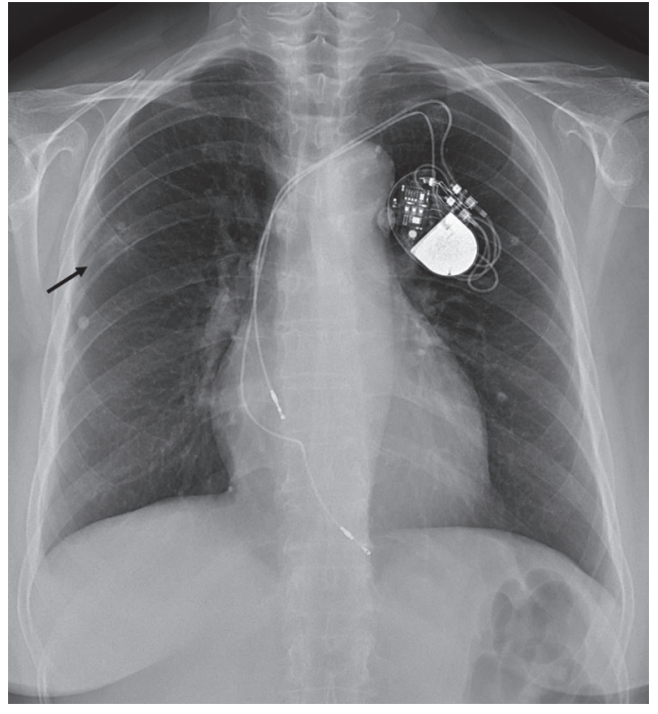

Figure 1 The initial chest radiograph. The arrow indicates nodular opacity in the right upper lung field. positron emission tomography (PET)/CT scan was performed; maximum standardized uptake value (SUVmax) of 2.7 was obtained in the two subsolid nodules in the RUL (Figure 3). Moreover, high metabolic uptake (SUVmax of 10.6) was noted in the left supraclavicular, bilateral hilar and mediastinal, and periportal areas and the sacrum. Due to marked discrepancy in the SUVmax between the lung nodules and lymph nodes or extra-thoracic lesion, lymphoma and other systemic inflammatory diseases were considered in the differential diagnosis. First, fine needle aspiration was performed in the left supraclavicular lymph node, which revealed benign findings. Next, to confirm the diagnosis, thoracoscopic wedge resection was performed for the larger nodule in the RUL. Pathologic examination finding was non-caseating epithelioid granulomas that were negative for Mycobacterium tuberculosis and non-tuberculosis mycobacterium, as assessed using the polymerase chain reaction (PCR) test performed on the biopsy tissue,
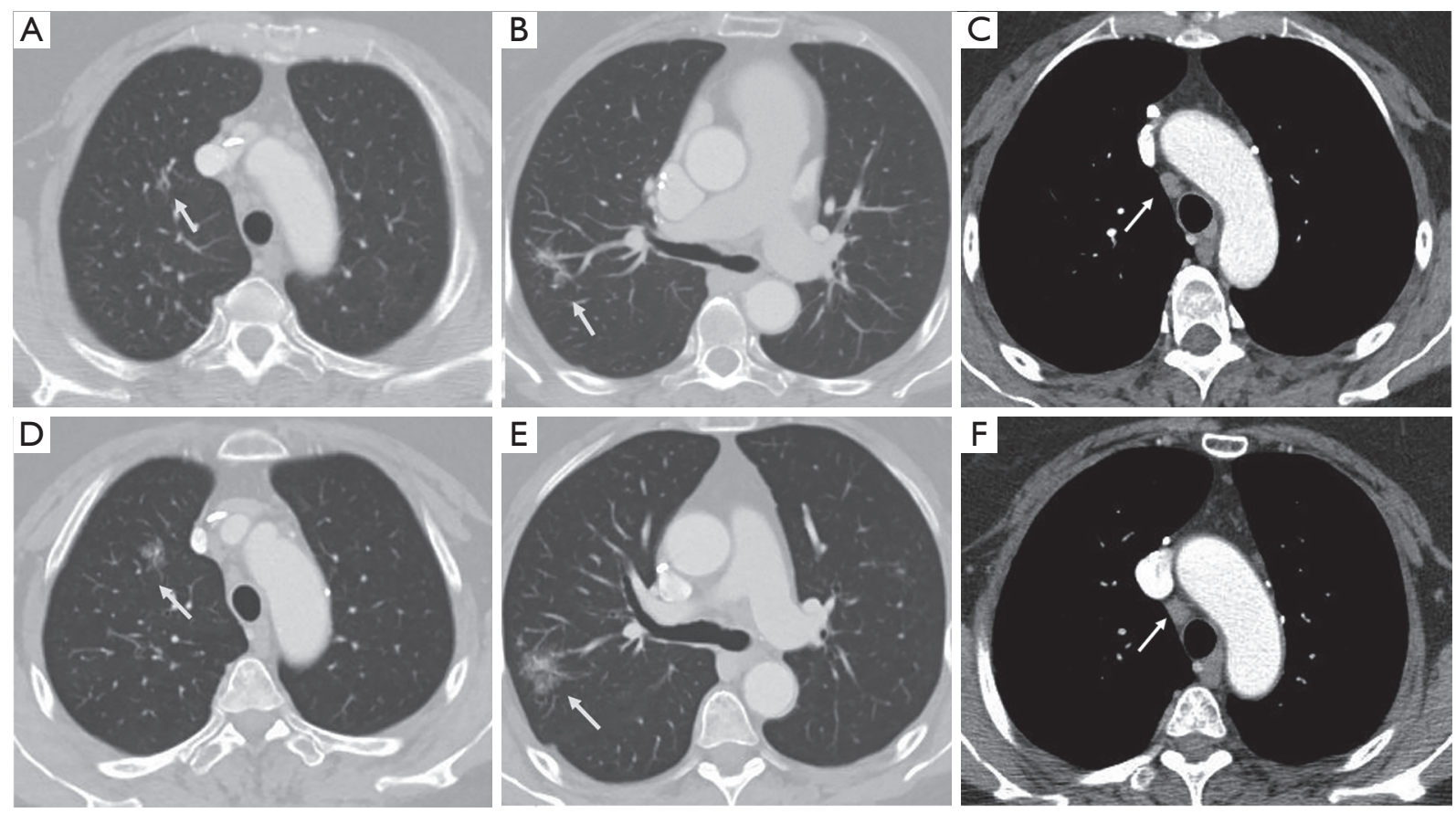

Figure 2 Chest computed tomography (CT) scan shows the presence of two subsolid nodules (white arrows) in the right upper lobe (A,B) and benign-looking mediastinal lymph nodes enlargement (C: white arrow). Follow-up chest CT scan taken after 15 months shows slow interval growth of the nodules (D,E: white arrows) without change in size of mediastinal lymph nodes (F: white arrow). 
and without evidence of other granulomatous diseases (Figure 4). Sputum acid fast stain and culture, PCR for Mycobacterium species, and culture for fungi were all negative. Angiotensin-converting enzyme levels were within normal range at $46.3 \mathrm{IU} / \mathrm{L}$ (reference 18-55 IU/L). Tests for anti-nuclear antibodies, anti-neutrophil cytoplasmic

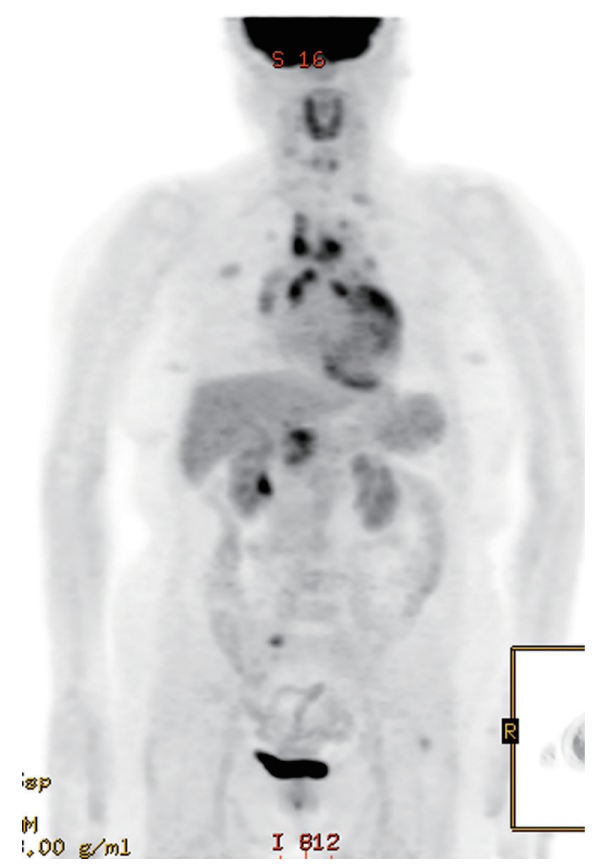

Figure 3 Positron emission tomography maximal intensity projection indicates abnormal hypermetabolism in the two partsolid nodules of the right upper lobe. Moreover, high metabolic uptake is observed in the left supraclavicular, bilateral hilar and mediastinal, and periportal areas and the sacrum. antibodies, and rheumatoid factor were negative. Our patient met the three criteria for diagnosis of sarcoidosis including clinical and radiological presentation, histological evidence of non-caseating granulomas, and evidence of no alternative diseases (1); ultimately, sarcoidosis involving the lung, multiple lymph nodes, and bone was determined, and sarcoidosis was considered as cause of her previous uveitis and AV block. Despite absence of symptoms, the patient was treated with oral prednisolone at initial dose of $0.5 \mathrm{mg} / \mathrm{kg}$ followed by gradual tapering for 3 months to determine the therapeutic response. The remnant nodular lesion in the RUL after surgical resection was nearly resolved and mediastinal and hilar lymph nodes decreased on the follow-up chest CT scan (Figure $5 A, B$ ).

\section{Discussion}

Sarcoidosis is a systemic disease of unknown cause characterized by non-caseating epithelioid granulomas in various organs $(1,2)$. The clinical presentation and natural history of sarcoidosis are highly variable (1). Some patients have symptoms suddenly followed by improvement in a short period of time, while others have symptoms that develop more gradually and last over a long period of time. Herein, we report a case of sarcoidosis that was diagnosed about 20 years after initial isolated extra-thoracic manifestation occurred as well as that presented with pulmonary subsolid nodules.

Pulmonary nodular sarcoidosis is an uncommon form of sarcoidosis and can be often misdiagnosed as lung cancer (5-7). In particular, pulmonary sarcoidosis may present as subsolid nodules when the patchy ground-glass opacities are
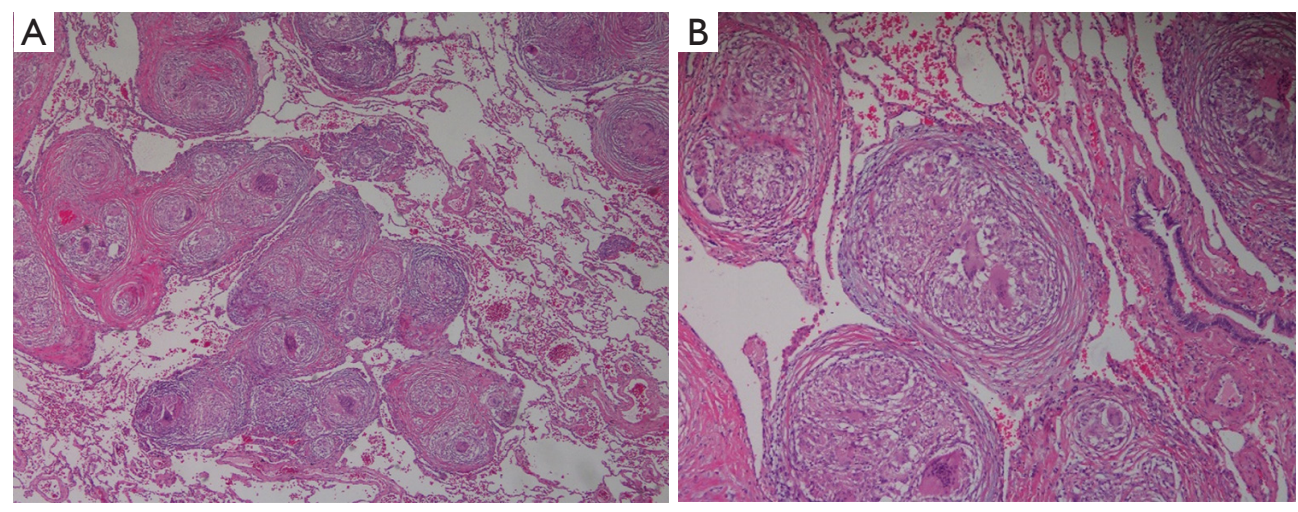

Figure 4 Pathologic examination of the lung specimen through thoracoscopic wedge resection reveals non-caseating epithelioid granulomas [hematoxylin and eosin stain, $\times 100(\mathrm{~A})$ and $\times 400(\mathrm{~B})]$. 

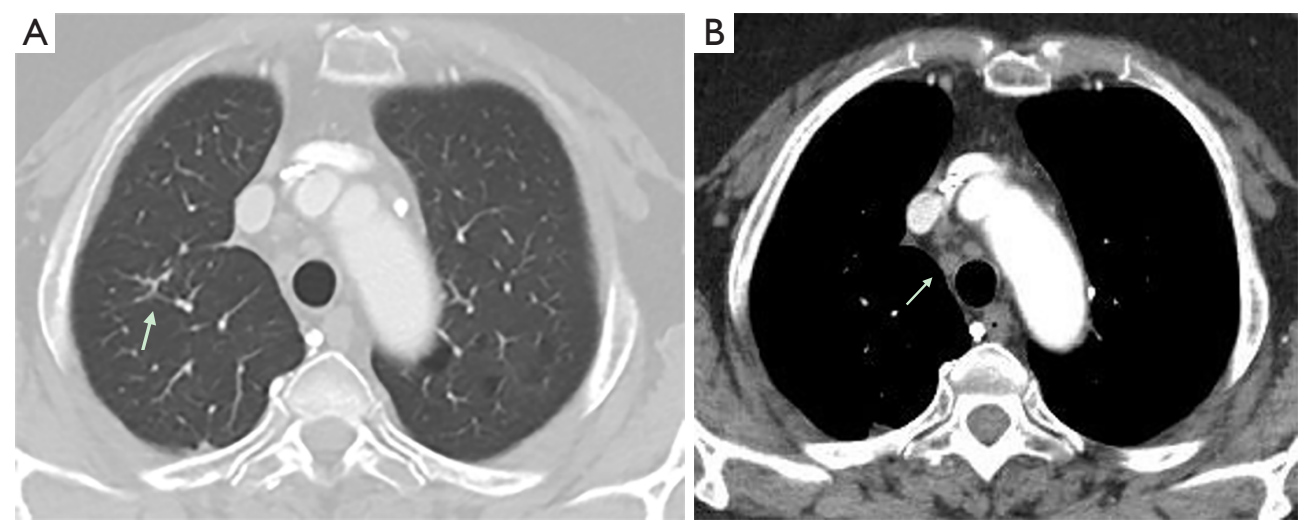

Figure 5 Follow-up chest computed tomography scan shows that the remnant nodular lesion (arrow) in the right upper lobe after surgical resection was nearly resolved (A) and mediastinal lymph nodes (arrow) decreased (B).

superimposed on a background of interstitial nodules $(3,4)$. Subsolid nodules persisting throughout follow-up generally represent a spectrum of diseases from atypical adenomatous hyperplasia to invasive adenocarcinoma; in such cases, the diagnosis of sarcoidosis is fairly challenging without a high index of clinical suspicion (8).

Lung cancers appearing as subsolid nodules generally have earlier stages than solid lung cancers (9); therefore, the pulmonary lesions suspected as early stage lung adenocarcinoma in our case may not be accompanied with metastases to multiple lymph nodes or other organs. Thus, in cases with peripheral subsolid nodules, PET/CT findings of involvement of multiple organs favor the diagnosis of sarcoidosis or lymphoma rather than that of advanced lung cancer.

Sarcoidosis typically involves the lungs, but up to $30 \%$ of patients present with extra-pulmonary sarcoidosis as the initial manifestation $(10,11)$. Our patient had a history of panuveitis followed by complete AV block of unknown cause, which was later presumably attributed to sarcoidosis. Past medical records related to her uveitis were unavailable, whereas those of her cardiac manifestation 10 years ago were available for review: she presented with chest pain and dizziness at our emergency department; complete $\mathrm{AV}$ block and ST elevation in V1-V3 were found on the initial electrocardiogram, but cardiac enzyme levels, echocardiography, and coronary angiography were all normal; thus, she underwent implantation of a permanent pacemaker without definite cause revealed. Isolated extra-thoracic lesion may result in unlikely suspicion of sarcoidosis. Moreover, definitive diagnosis of cardiac sarcoidosis is problematic due to difficulty in obtaining histological proof $(10,12)$. However, an earlier diagnosis of sarcoidosis could have been achieved in this case, if the attending clinician had paid more attention to the history of uveitis with uncertain cause.

In addition, the lymph nodes in the supraclavicular and periportal areas, as well as the sacrum are not the commonly affected sites of sarcoidosis. The findings of multiple atypical radiologic patterns including pulmonary subsolid nodules, lymphadenopathy at unusual locations, and focal bone involvement contributed to the delayed diagnosis of sarcoidosis. Previous history of uveitis or AV block of uncertain cause may be helpful in differentiating between sarcoidosis and lymphoma in such cases.

In conclusion, this case highlighted that atypical pulmonary manifestation as well as isolated extra-thoracic presentation may result in delayed diagnosis of sarcoidosis. The involvement of multiple organs favors diagnosis of sarcoidosis over that of advanced malignancy in cases with pulmonary subsolid nodule. Clinicians should consider previous history of extra-thoracic involvement of possible sarcoidosis, such as uveitis or AV block, as useful information to enable diagnosis.

\section{Acknowledgments}

None.

\section{Footnote}

Conflicts of Interest: The authors have no conflicts of interest to declare.

Ethical Statement: The authors are accountable for all 
aspects of the work in ensuring that questions related to the accuracy or integrity of any part of the work are appropriately investigated and resolved. Written informed consent was obtained from the patient for publication of this case report.

\section{References}

1. Statement on sarcoidosis. Joint Statement of the American Thoracic Society (ATS), the European Respiratory Society (ERS) and the World Association of Sarcoidosis and Other Granulomatous Disorders (WASOG) adopted by the ATS Board of Directors and by the ERS Executive Committee, February 1999. Am J Respir Crit Care Med 1999;160:736-55.

2. Baughman RP, Lower EE, du Bois RM. Sarcoidosis. Lancet 2003;361:1111-8.

3. Criado E, Sanchez M, Ramirez J, et al. Pulmonary sarcoidosis: typical and atypical manifestations at highresolution CT with pathologic correlation. Radiographics 2010;30:1567-86.

4. Park HJ, Jung JI, Chung MH, et al. Typical and atypical manifestations of intrathoracic sarcoidosis. Korean J Radiol 2009;10:623-31.

5. Sweidan AJ, Singh NK, Stein A, et al. Nodular Sarcoidosis

Cite this article as: Lee HJ, Seo H, Cha SI, Park TI, Kim CH, Lee J. Sarcoidosis presenting pulmonary subsolid nodules that mimic lung adenocarcinoma in a patient with history of uveitis and arrhythmia: a case report. Ann Transl Med 2019;7(18):496. doi: $10.21037 /$ atm.2019.08.98
Masquerading as Cancer. Clin Med Insights Circ Respir Pulm Med 2017;11:1179548417703123.

6. Malaisamy S, Dalal B, Bimenyuy C, et al. The clinical and radiologic features of nodular pulmonary sarcoidosis. Lung 2009;187:9-15.

7. Lee HN, Kim JI, Won K, et al. Atypical CT findings of pulmonary sarcoidosis: A case report. Medicine (Baltimore) 2018;97:e11456.

8. Henschke CI, Yankelevitz DF, Mirtcheva R, et al. CT screening for lung cancer: frequency and significance of part-solid and nonsolid nodules. AJR Am J Roentgenol 2002;178:1053-7.

9. Noguchi M, Morikawa A, Kawasaki M, et al. Small adenocarcinoma of the lung. Histologic characteristics and prognosis. Cancer 1995;75:2844-52.

10. West SG. Current management of sarcoidosis I: pulmonary, cardiac, and neurologic manifestations. Curr Opin Rheumatol 2018;30:243-8.

11. Newman LS, Rose CS, Maier LA. Sarcoidosis. N Engl J Med 1997;336:1224-34.

12. Sayah DM, Bradfield JS, Moriarty JM, et al. Cardiac Involvement in Sarcoidosis: Evolving Concepts in Diagnosis and Treatment. Semin Respir Crit Care Med 2017;38:477-98. 